\title{
Erratum to: Pili contribute to biofilm formation in vitro in Mycobacterium tuberculosis
}

\author{
Saiyur Ramsugit - Sinenhlanhla Guma • Balakrishna Pillay • \\ Paras Jain · Michelle H. Larsen • Siva Danaviah • \\ Manormoney Pillay
}

Published online: 6 September 2013

(C) Springer Science+Business Media Dordrecht 2013

\section{Erratum to: Antonie van Leeuwenhoek DOI 10.1007/s10482-013-9981-6}

In the original published version, Tables 1 and 2 are incorrect. The correct version of Tables 1 and 2 is given here:

The online version of the original article can be found under doi:10.1007/s10482-013-9981-6.

S. Ramsugit · M. Pillay $(\bowtie)$

Medical Microbiology and Infection Control, School of Laboratory Medicine and Medical Sciences, College of Health Sciences, University of KwaZulu-Natal, 1st Floor Doris Duke Medical Research Institute, Congella, Private Bag 7, Durban 4013, South Africa

e-mail: pillayc@ukzn.ac.za

S. Guma · B. Pillay

Microbiology, University of KwaZulu-Natal, Durban, South Africa

P. Jain

Department of Microbiology and Immunology, Albert Einstein College of Medicine, New York, NY, USA

P. Jain - M. H. Larsen

Howard Hughes Medical Institute, Albert Einstein

College of Medicine, New York, NY, USA
M. H. Larsen

Department of Medicine, Albert Einstein College of Medicine, New York, NY, USA

S. Danaviah

Africa Centre for Health and Population Studies, University of KwaZulu-Natal, Durban, South Africa 
Table 1 Primers and probes used in this study

\begin{tabular}{|c|c|c|c|}
\hline Name $^{a}$ & Purpose & Primer/probe sequence ${ }^{b}$ & $\begin{array}{l}\text { Product } \\
\text { size (bp) }\end{array}$ \\
\hline $\begin{array}{l}\text { Mtp-LL } \\
\text { Mtp-LR }\end{array}$ & $\begin{array}{l}\text { Upstream allelic } \\
\text { exchange substrate } \\
\text { primers }\end{array}$ & $\begin{array}{l}\text { 5'-TTTTTTTTGCATAAATTGCTCACGATTCATGCCGGTGAG-3' }^{\prime} \\
\text { 5'-TTTTTTTGCATTTCTTGCCGCCACTGTGAATCAGGTGTGA }^{\prime} \\
\text { TGTCTCACTGAGGTCTCTGTTTGGGCTGCGGACTGC-3' }\end{array}$ & 731 \\
\hline $\begin{array}{l}\text { Mtp-RL } \\
\text { Mtp-RR }\end{array}$ & $\begin{array}{l}\text { Downstream allelic } \\
\text { exchange substrate } \\
\text { primers }\end{array}$ & $\begin{array}{l}\text { 5'-TTTTTTTTCCATAGATTGGGGGCTGTGTTAGCACTCCTACG }^{\text {AGTGTCTGGTCTCGTAGAAGGTCCCGTGCTTGACGAT-3' }} \\
\text { 5'-TTTTTTTTCCATCTTTTGGAAATTGCGTCCCGCTTGAAA-3' }\end{array}$ & 776 \\
\hline $\begin{array}{l}\text { Mtp-1F } \\
\text { Mtp-1R }\end{array}$ & $\begin{array}{l}\text { Amplification of region } \\
\text { containing } m t p \text { gene }\end{array}$ & $\begin{array}{l}\text { 5'-GATCGCTTTCGTACCGTCAT-3' } \\
\text { 5'-ACGCGTATTCGCTGATGC-3' }\end{array}$ & 370 \\
\hline $\begin{array}{l}\text { Mtp-2F } \\
\text { Mtp-2R }\end{array}$ & $\begin{array}{l}\text { Amplification of region } \\
\text { containing } m t p \text { gene }\end{array}$ & $\begin{array}{l}5^{\prime} \text {-CTCATGGGTCACAGCGAGTA-3' } \\
\text { 5'-ATGACAGGTTCCCTTCAAGC-3' }^{\prime}\end{array}$ & 583 \\
\hline $\begin{array}{l}\text { Hyg-F } \\
\text { Hyg-R }\end{array}$ & $\begin{array}{l}\text { Amplification of region } \\
\text { of } h y g^{\mathrm{R}} \text { cassette }\end{array}$ & $\begin{array}{l}\text { 5'-ACCCCCCATTCCGAGGTCTT-3' } \\
\text { 5'-CCGGAAGGCGTTGAGATGCA-3' }^{\prime}\end{array}$ & 300 \\
\hline $\begin{array}{l}\text { INS-1 } \\
\text { INS-2 }\end{array}$ & $\begin{array}{l}\text { Amplification of MTB's } \\
\text { insertion sequence } \\
\text { IS6110 }\end{array}$ & $\begin{array}{l}5^{\prime} \text {-CGTGAGGGCATCGAGGTGGC-3' } \\
\text { 5'-GCGTAGGCGTCGGTGACAAA-3' }^{\prime}\end{array}$ & 245 \\
\hline $\begin{array}{l}\text { SH-F } \\
\text { SH-R }\end{array}$ & $\begin{array}{l}\text { Generation of probe for } \\
\text { Southern hybridization }\end{array}$ & 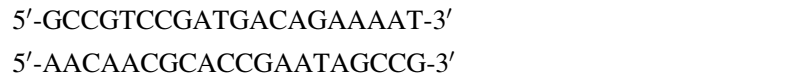 & 783 \\
\hline $\begin{array}{l}\text { Mtp-261F } \\
\text { Mtp-261R }\end{array}$ & $\begin{array}{l}\text { Amplification of } m t p \\
\text { gene for insertion into } \\
\text { pMV261 for } \\
\text { complementation }\end{array}$ & $\begin{array}{l}5^{\prime} \text {-TTTTTAAGATCTCATGTACCGGTTCGCGTG-3' } \\
\text { 5'-TTTTTTAAGCTTGGCACGGGAGCGTAAATCTG-3' }\end{array}$ & 436 \\
\hline $\begin{array}{l}\text { Mtp-AnyF } \\
\text { Mtp-AnyR } \\
\text { mtp probe }\end{array}$ & $\begin{array}{l}\text { Primers and probe for } \\
\text { detection of } m t p \\
\text { expression in } \mathrm{qPCR}\end{array}$ & $\begin{array}{l}\text { 5'-CCCAACTGGGATCCCTACAC-3' } \\
\text { 5'-AGTCGCGGCTGTGGTC-3' } \\
\text { FAM-CTGCCATGACGACTTC-MGBNFQ }\end{array}$ & 73 \\
\hline $\begin{array}{l}16 \mathrm{~S}-\text { AnyF } \\
16 \mathrm{~S}-\text { AnyR } \\
16 \mathrm{~S} \text { probe }\end{array}$ & $\begin{array}{l}\text { Primers and probe for } \\
\text { detection of } 16 \mathrm{~S} \text { rRNA } \\
\text { expression in qPCR }\end{array}$ & $\begin{array}{l}\text { 5'-GGGTGACGGCCTACCAA-3' } \\
\text { 5'-GCCGGACACCCTCTCA-3' }^{\prime} \\
\text { FAM-CCGGCTACCCGTCGTC-MGBNFQ }\end{array}$ & 54 \\
\hline
\end{tabular}

${ }^{\mathrm{a}}$ Forward primer listed above the reverse primer for each primer pair

${ }^{\mathrm{b}}$ Restriction sites are underlined

Table 2 Bioinformatics analysis of the $m t p$ translation product

\begin{tabular}{|c|c|}
\hline Property & Result $^{\mathrm{a}}$ \\
\hline $\begin{array}{l}\text { Amino acid } \\
\text { sequence }\end{array}$ & $\begin{array}{l}\text { MYRFACRTLMLAACILATGVAGLGVGAQSAAQTAPVPDYYWCPGQPFDPAWGPNWDPYTCHDDFHRDSDGP } \\
\text { DHSRDYPGPILEGPVLDDPGAAPPPPAAGGGA }\end{array}$ \\
\hline $\begin{array}{l}\text { Transmembrane } \\
\text { analysis }\end{array}$ & $\begin{array}{l}\text { iiiiiiittttttttttttttttt } 000000000000000000000000000000000000000000000 \\
00000000000000000000000000000000\end{array}$ \\
\hline Signal peptide & 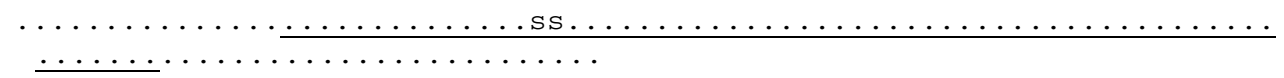 \\
\hline $\begin{array}{r}\text { Secondary } \\
\text { structure }\end{array}$ & 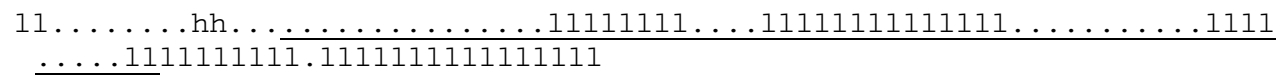 \\
\hline $\begin{array}{l}\text { Protein-binding } \\
\text { capacity }\end{array}$ & 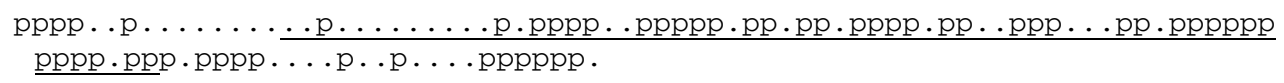 \\
\hline Hydrophobicity & $\begin{array}{l}+++++++++++++++\underline{+}++++++++++++++---++-------------------------- \\
\quad \underline{--------------------++++--++-----+-------}\end{array}$ \\
\hline
\end{tabular}

